\title{
Selective Probing of Hidden Spin-Polarized States in Inversion-Symmetric Bulk $\mathrm{MoS}_{2}$
}

\author{
E. Razzoli, ${ }^{1, *}$ T. Jaouen, ${ }^{1}$ M.-L. Mottas, ${ }^{1}$ B. Hildebrand, ${ }^{1}$ G. Monney, ${ }^{1}$ A. Pisoni, ${ }^{2}$ S. Muff, ${ }^{3,4}$ M. Fanciulli, ${ }^{3,4}$ N. C. Plumb, ${ }^{3}$ \\ V. A. Rogalev, ${ }^{3}$ V. N. Strocov, ${ }^{3}$ J. Mesot, ${ }^{3,4,5}$ M. Shi, ${ }^{3}$ J. H. Dil,${ }^{3,4}$ H. Beck, ${ }^{1}$ and P. Aebi ${ }^{1}$ \\ ${ }^{1}$ Département de Physique and Fribourg Center for Nanomaterials, Université de Fribourg, CH-1700 Fribourg, Switzerland \\ ${ }^{2}$ Laboratory of Physics of Complex Matter, Ecole Polytechnique Fédérale de Lausanne, CH-1015 Lausanne, Switzerland \\ ${ }^{3}$ Swiss Light Source, Paul Scherrer Institute, CH-5232 Villigen PSI, Switzerland \\ ${ }^{4}$ Institute of Physics, École Polytechnique Fédérale de Lausanne, CH-1015 Lausanne, Switzerland \\ ${ }^{5}$ Laboratory for Solid State Physics, ETH Zürich, CH-8093 Zürich, Switzerland
}

(Received 25 November 2016; published 22 February 2017)

\begin{abstract}
Spin- and angle-resolved photoemission spectroscopy is used to reveal that a large spin polarization is observable in the bulk centrosymmetric transition metal dichalcogenide $\mathrm{MoS}_{2}$. It is found that the measured spin polarization can be reversed by changing the handedness of incident circularly polarized light. Calculations based on a three-step model of photoemission show that the valley and layer-locked spinpolarized electronic states can be selectively addressed by circularly polarized light, therefore providing a novel route to probe these hidden spin-polarized states in inversion-symmetric systems as predicted by Zhang et al. [Nat. Phys. 10, 387 (2014).].
\end{abstract}

DOI: 10.1103/PhysRevLett.118.086402

Transition metal dichalcogenide (TMDC) monolayers have been heavily investigated due to the locking of the spin with valley pseudospins and the presence of a direct gap, which makes them ideal candidates for valleytronic devices [1]. Thanks to the lack of inversion symmetry and the non-negligible spin-orbit coupling, TMDC monolayers also feature well-defined spin-polarized ground states [2], which can, in principle, be investigated by spin- and angleresolved photoemission spectroscopy (spin-ARPES). However, while few ARPES studies clearly observed the indirect to direct band gap transition going from the bulk crystal to the monolayer [3-6], spin-ARPES investigation of TMDC monolayers is more challenging, given the low cross section of photoemission from single layers.

ARPES measurements on the bulk system are instead less demanding, but early studies detected spin-resolved signals only from TMDCs with broken inversion symmetry [7]. Interestingly, a recent theoretical study [8] suggested that the spin texture of the TMDC could be probed by photoemission, even in the inversion symmetric bulk TMDC crystals, as a result of the localization of two spin-degenerated valence band maxima on different layers of the unit cell and of the finite penetration depth of the photoemission process probing preferentially the uppermost layer. Experimentally this effect has been observed for $\mathrm{WSe}_{2}$, where the spin orbit (SO) coupling is the strongest one among the TMDCs [9].

Here we show that a large out-of-plane spin-polarization is observable in the bulk dichalcogenide $\mathrm{MoS}_{2}$, and more importantly, that its sign depends on the handedness of the incident circularly polarized light. Our calculations, based on a three step model of the photoemission process demonstrate that the observed spin reversal is an initial state effect. Using left- $\left(C_{L}\right)$ and right-handed $\left(C_{R}\right)$ circularly polarized light results in selecting different initial states that present a positive or negative out-of-plane spin polarization depending on their localization on the S-Mo-S layers of the $2 \mathrm{H}$-stacked $\mathrm{MoS}_{2}$ unit cell. Our findings not only highlight the locking of the spin with layer and valley pseudospins in $\mathrm{MoS}_{2}$ but also provide a novel and improved route, other than taking profit of the inelastic mean free path (IMFP) [8], for selectively probing hidden spin-polarized bands in inversion symmetric systems.

The soft $x$-ray ARPES and spin-ARPES experiments were, respectively, performed at the ADRESS beam line [10] and at the COPHEE end station of SIS beam line at the Swiss Light Source, Paul Scherrer Institut, Villigen, Switzerland. Base pressures were better than $4 \times 10^{-10} \mathrm{mbar}$, with a sample temperature of $\sim 25 \mathrm{~K}$ [11]. The chemical vapor transport-grown $2 \mathrm{H}$ - $\mathrm{MoS}_{2}$ crystal consisting of hexagonal $2 \mathrm{H}$-stacked S-Mo-S layers separated by a van der Waals gap [Fig. 1(a) [18]], has been cleaved in situ at low temperature. Figure 1(b) (top panel) shows the ARPES intensity plot of bulk $\mathrm{MoS}_{2}$ along the $\bar{K}-\bar{\Gamma}$ high-symmetry direction of the Brillouin zone [bottom of Fig. 1(a)] and measured using circularly polarized light with an overall energy resolution of 50-80 meV. The observed bands are in agreement with previous ARPES measurements [3,5,19], with the top of the valence band at $\bar{\Gamma}$ originating from Mo $d_{z^{2}} / p_{z}$ orbitals and that lies $\sim 600$ and $\sim 800 \mathrm{meV}$ above the upper (VB1) and lower (VB2) valence bands at $\bar{K}$ with mainly Mo $d_{x^{2}-y^{2}}, d_{x y}$ orbital characters. The observed dispersion is in good agreement with our density functional theory (DFT) calculation within the local density approximation (LDA) between $\Gamma$ and $K$, given the small $k_{z}$ 

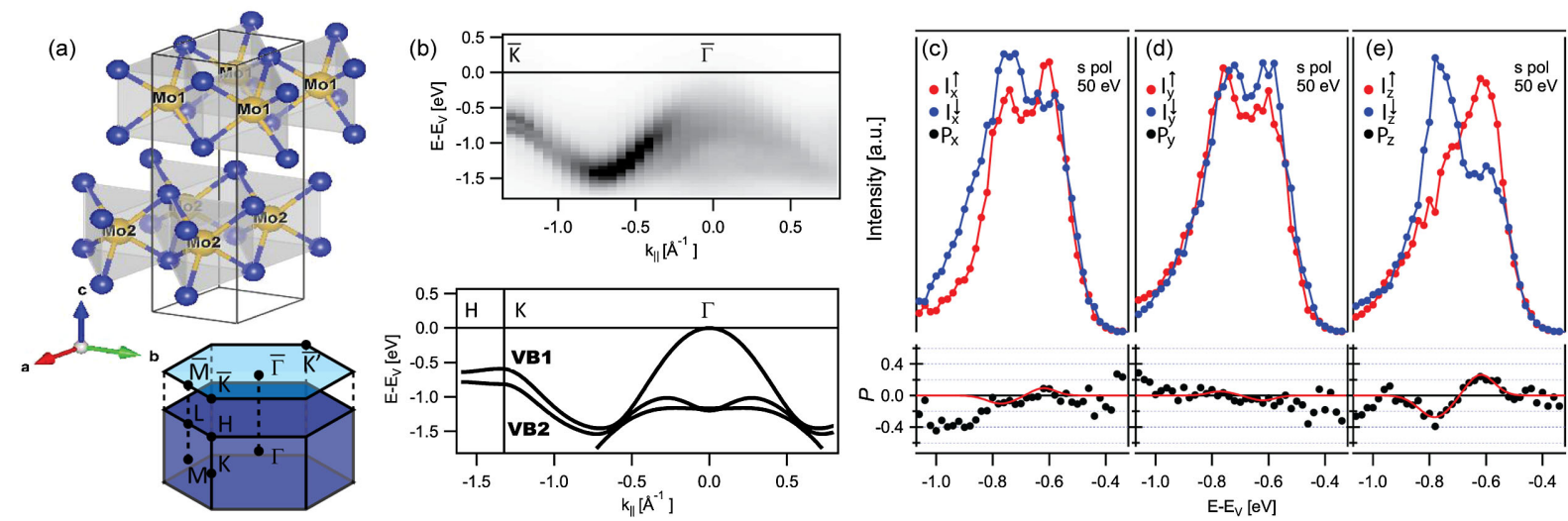

FIG. 1. (a) Structural model (top) and Brillouin zone (bottom) of $2 H-\mathrm{MoS}_{2}$. (b) ARPES intensity map along $\bar{K}-\bar{\Gamma}$ direction (top), and DFT-LDA calculated band structure showing the band dispersion along $K-\Gamma$ and $H-K$ directions (bottom). (c)-(d) Spin-resolved intensities (top) and spin polarization curves (bottom) at the $\bar{K}$ point along $x, y$, and $z$, respectively. Continuous red lines in the bottom panels are Gaussian fits, resulting in $P_{x}=\mp 0.08, P_{y}= \pm 0.05$, and $P_{z}=\mp 0.28$. Spin-ARPES data were acquired at $h \nu=50 \mathrm{eV}$ with $s$-polarized light.

dispersion along the $K-H$ direction [see Fig. 1(b) bottom panel] [11].

Figures 1(c)-1(e) present the experimental spin-ARPES intensities (top) and the spin-polarization energy distribution curves (bottom) at the $\bar{K}$ point along the three spatial coordinates $x, y$, and $z$ in the sample frame measuring inplane $\left(P_{x}, P_{y}\right)$, and out-of-plane $\left(P_{z}\right)$ spin polarization. Conversion from the coordinate system given by the Mott polarimeters to the sample frame is performed according to Ref. [20]. The difference in binding energy between VB1 and VB2 is $170 \mathrm{meV}$, in agreement with previous studies [19]. The measured spin polarization is almost entirely outof-plane with a negligible small in-plane component. The measured out-of-plane spin polarization points upwards and downwards for the upper and lower valence band, respectively, similarly to what has been observed in $\mathrm{WSe}_{2}$ [9].

The $z$ component of the spin polarization displays an interesting polarization dependence [Fig. 2]. The spin polarization measured with $p$ [Fig. 2(a)], and $C_{L}$ polarization [Fig. 2(b)] are in agreement with the observation with $s$-polarized light [Fig. 1(e)], with the only difference of an increasing absolute value of the spin polarization fraction. Instead, when probed with $C_{R}$ light the out-ofplane spin polarization changes its sign; i.e., the $z$ component of the detected photoelectron points upwards and downwards for the lower and upper valence band, respectively [Fig. 2(c)]. Interestingly, the light handednessinduced reversal of the $z$ component is also observable at other photon energy [e.g., at $h \nu=58 \mathrm{eV}$ as shown in
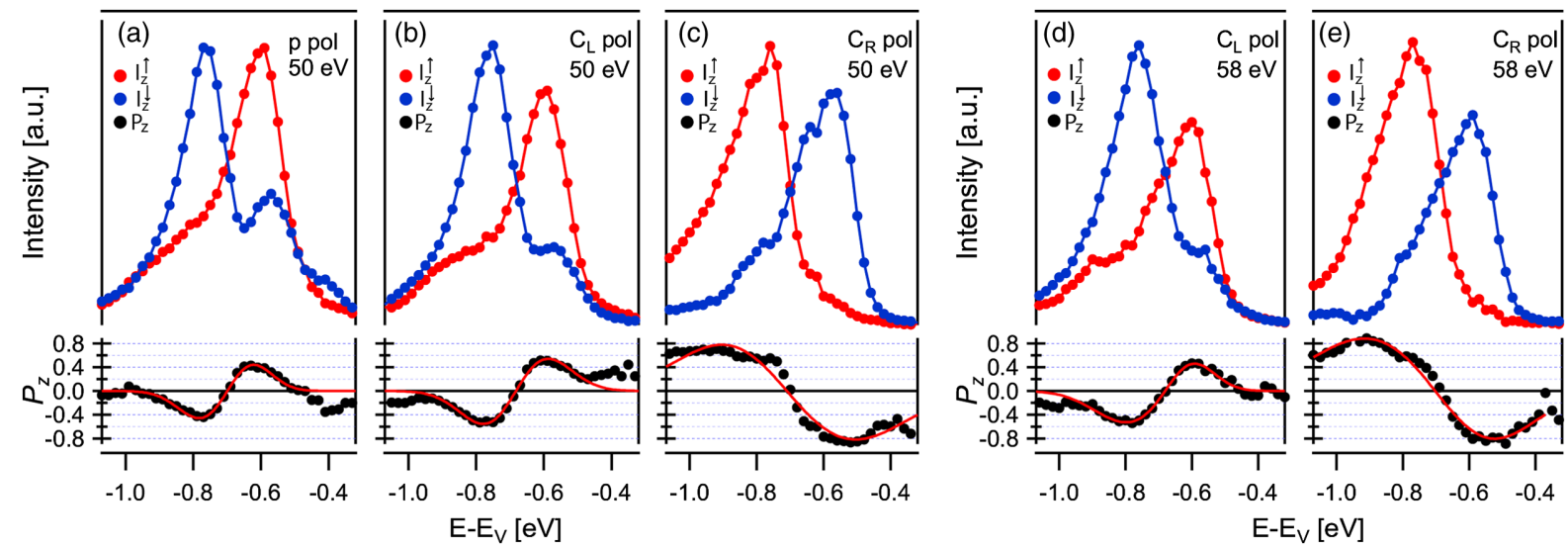

FIG. 2. Spin-ARPES data acquired at $h \nu=50$ and $h \nu=58 \mathrm{eV}$. (a)-(c) Spin-resolved intensities (top) and spin polarization curves (bottom) along $z$ at the $\bar{K}$ point acquired with $p, C_{L}$, and $C_{R}$ polarization, respectively $(h \nu=50 \mathrm{eV})$. (d),(e) Spin-resolved intensities (top) and spin polarization curves (bottom) at the $\bar{K}$ point acquired with $C_{L}$ and $C_{R}$ polarization, and $h \nu=58 \mathrm{eV}$. Continuous red lines in the bottom panels are Gaussian fits, resulting in $P_{z}(p, 50 \mathrm{eV})=\mp 0.50, P_{z}\left(C_{L}, 50 \mathrm{eV}\right)=\mp 0.68, P_{z}\left(C_{R}, 50 \mathrm{eV}\right)= \pm 0.85$, $P_{z}\left(C_{L}, 58 \mathrm{eV}\right)=\mp 0.54$, and $P_{z}\left(C_{R}, 58 \mathrm{eV}\right)= \pm 0.91$. 
Figs. 2(d) and 2(e)], therefore indicating that it does not depend on the three-dimensional $\left(k_{z}\right)$ band dispersion.

A possible explanation of our observation would be that photoelectron spin flips during the photoemission process; i.e., we observed a final state matrix element effect. Up to now, most of the spin-ARPES studies dealing with $k$-resolved light-induced spin rotations have been carried out on topological insulators (TI), due to the large strength of the SO coupling and to the high quality of the ARPES data in such systems [21-24]. It has been experimentally shown that light-induced spin rotations are observable only at low photon energy (about $6 \mathrm{eV}$ ) $[21,24]$. Theoretically, it has been suggested that the SO-induced term in the lightmatter interaction Hamiltonian $H_{\text {SO }}^{\text {int }}[25]$ is responsible for the spin events tuned by light polarization. However, in contrast to the TI that exhibit extremely large SO coupling, in TMDCs and especially in $\mathrm{MoS}_{2}[5]$, the $\left\langle i\left|H_{\text {SO }}^{\text {int }}\right| f\right\rangle$ term can be neglected and the observed flip in the out-of-plane spin polarization has to be related to a different mechanism [11].

Instead, the origin of the sign inversion of $P_{z}$ can be understood analyzing the idealized case of a TMDC bilayer with small interlayer coupling $t_{\perp}$. In this simplified model, the top of the valence band VB1 $\left(E_{b}=E_{1}\right)$ at $K$ is doubly degenerate and originates in Mo $d_{x^{2}-y^{2}}, d_{x y}$ orbitals. The eigenstates expressed in terms of spherical harmonics $\left|l, m_{\mu}\right\rangle$ are then given by $[11,26]$

$$
\begin{aligned}
\left|1, E_{1}\right\rangle & =\frac{\cos \alpha|2,2\rangle_{1}+\sin \alpha|2,-2\rangle_{2}}{\sqrt{2}}|\uparrow\rangle, \\
\left|2, E_{1}\right\rangle & =\frac{\sin \alpha|2,2\rangle_{1}+\cos \alpha|2,-2\rangle_{2}}{\sqrt{2}}|\downarrow\rangle,
\end{aligned}
$$

(a)

(b)
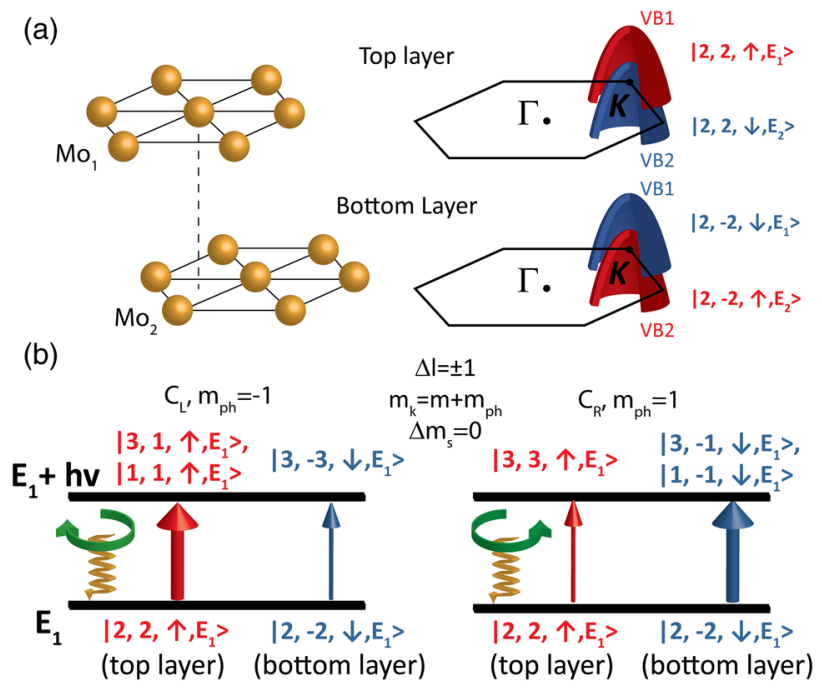

FIG. 3. (a) Schematic of the layer-resolved band dispersion of VB1 and VB2 around the $K$ point. (b) Dipole-allowed transitions for the eigenstate of VB1 for vanishing interlayer coupling $(\alpha=0)$ at the $K$ point. using $\mu=1$ (2) for the upper (lower) Mo layer of the $2 \mathrm{H}$-stacked $\mathrm{MoS}_{2}$ unit cell [Fig. 3(a)], and with $\cos 2 \alpha=\lambda_{\mathrm{SO}} / \sqrt{\lambda_{\mathrm{SO}}^{2}+t_{\perp}^{2}}$ that corresponds to the spindependent layer polarization of the hole states. The spinvalley coupling of holes in monolayers $\lambda_{\mathrm{SO}}$ is calculated to be 1.7 times higher than the interlayer hopping $t_{\perp}$ in $\mathrm{MoS}_{2}$ [26], therefore resulting in a substantial layer-dependent spin polarization $(\cos 2 \alpha=0.863)$.

The states $\left|1, E_{1}\right\rangle$ and $\left|2, E_{1}\right\rangle$, respectively, contribute to the spin-up $\left(I_{\uparrow, z}^{\epsilon}\right)$ and spin-down $\left(I_{\downarrow, z}^{\epsilon}\right)$ intensity [11]:

$$
\begin{aligned}
& I_{\uparrow, z}^{\epsilon} \propto\left|\cos (\alpha) \boldsymbol{\kappa}_{2,2} \cdot \boldsymbol{\epsilon}+\sin (\alpha) \delta e^{-i \mathbf{k} \cdot \mathbf{d}_{l}} \boldsymbol{\kappa}_{2,-2} \cdot \boldsymbol{\epsilon}\right|^{2}, \\
& I_{\downarrow, z}^{\epsilon} \propto\left|\sin (\alpha) \boldsymbol{\kappa}_{2,2} \cdot \boldsymbol{\epsilon}+\cos (\alpha) \delta e^{-i \mathbf{k} \cdot \mathbf{d}_{l}} \boldsymbol{\kappa}_{2,-2} \cdot \boldsymbol{\epsilon}\right|^{2},
\end{aligned}
$$

with $\mathbf{k}_{l, m}=\left\langle e^{i \mathbf{k} \cdot \mathbf{r}}|\mathbf{r}| l, m\right\rangle, \mathbf{d}_{l}=\left(\frac{1}{2} a, \frac{2}{3} a,-\frac{1}{2} c\right)$ the relative atomic displacement between the upper and lower Mo layers, $\boldsymbol{\epsilon}$ the light polarization vector, and $\delta=e^{-\left(c / 2 \lambda_{e}\right)}$, an attenuation factor that takes into account the photoelectrons IMFP $\lambda_{e}$. For normal incident circularly polarized light the conservation of the total angular momentum dictates that only the states with $m_{k}=m \pm 1$ can give a nonvanishing contribution to $\boldsymbol{\kappa}_{l, m}$, once the free-electron final state is decomposed in spherical harmonics $\left|e^{i \mathbf{k} \cdot \mathbf{r}}\right\rangle=\sum_{l_{k}, m_{k}} d_{l_{k}, m_{k}}(\mathbf{k}, r)\left|l_{k}, m_{k}\right\rangle$.

In Fig. 3 we illustrate the dipole-allowed transitions for the eigenstate of VB1 at the $K$ point, for the case of vanishing interlayer coupling $(\alpha=0)$. In this case spin-up and spin-down states are fully locked on the upper and lower S-Mo-S layer, respectively [Fig. 3(a)].

For $C_{L}$ light [Fig. 3(b), left-hand side], the available final states have $m_{k}=m-1$, and are $\{|1,1\rangle,|3,1\rangle\}$ for $m=2$ and $\{|3,-3\rangle\}$ for $m=-2$. As expected from the higher number of final states available for a dipole transition from a state with $m=2$, our numerical evaluation shows that the cross section for transition to the states $\{|1,1\rangle,|3,1\rangle\}$ is 1 order of magnitude larger than to $\{|3,-3\rangle\}$, i.e., $\left|\boldsymbol{\kappa}_{2,2} \cdot \boldsymbol{\epsilon}_{C_{L}}^{\mathbf{n}}\right| /\left|\boldsymbol{\kappa}_{2,-2} \cdot \boldsymbol{\epsilon}_{C_{L}}^{\mathbf{n}}\right| \approx 10$ [11]. Similarly, and according to the relation $\boldsymbol{\kappa}_{l, \pm m} \cdot \boldsymbol{\epsilon}_{C_{R / L}}=\boldsymbol{\kappa}_{l, \mp m} \cdot \boldsymbol{\epsilon}_{C_{L / R}}$, the available final states of a dipole transition induced by $C_{R}$ light [Fig. 3(b) right-hand side], have $m_{k}=m+1$ and $\left|\boldsymbol{\kappa}_{2,-2} \cdot \boldsymbol{\epsilon}_{C_{R}}^{\mathbf{n}}\right| /\left|\boldsymbol{\kappa}_{2,2} \cdot \boldsymbol{\epsilon}_{C_{R}}^{\mathbf{n}}\right| \approx 10$. For a nonzero interlayer hopping, Eq. (2) thus gives

$$
P_{z}^{C_{R / L}^{\mathrm{n}}}=\frac{I_{\uparrow, z}^{\epsilon_{C_{R / L}}^{\mathrm{n}}}-I_{\downarrow, z}^{\epsilon_{C_{R / L}}^{\mathrm{n}}}}{\epsilon_{C_{R / L}}^{\mathrm{n}}+I_{\downarrow, z}^{\boldsymbol{n}_{C_{R / L}}}} \approx \mp \cos 2 \alpha .
$$

This demonstrates that the layer-locked, hidden spinpolarized states of the $K$ valley can be probed through the spin-dependent selection rules introduced by the use of circularly polarized light. This not only selects which hidden-spin polarized state will be observed, but also overcomes the IMFP-limited probing of the spin texture 

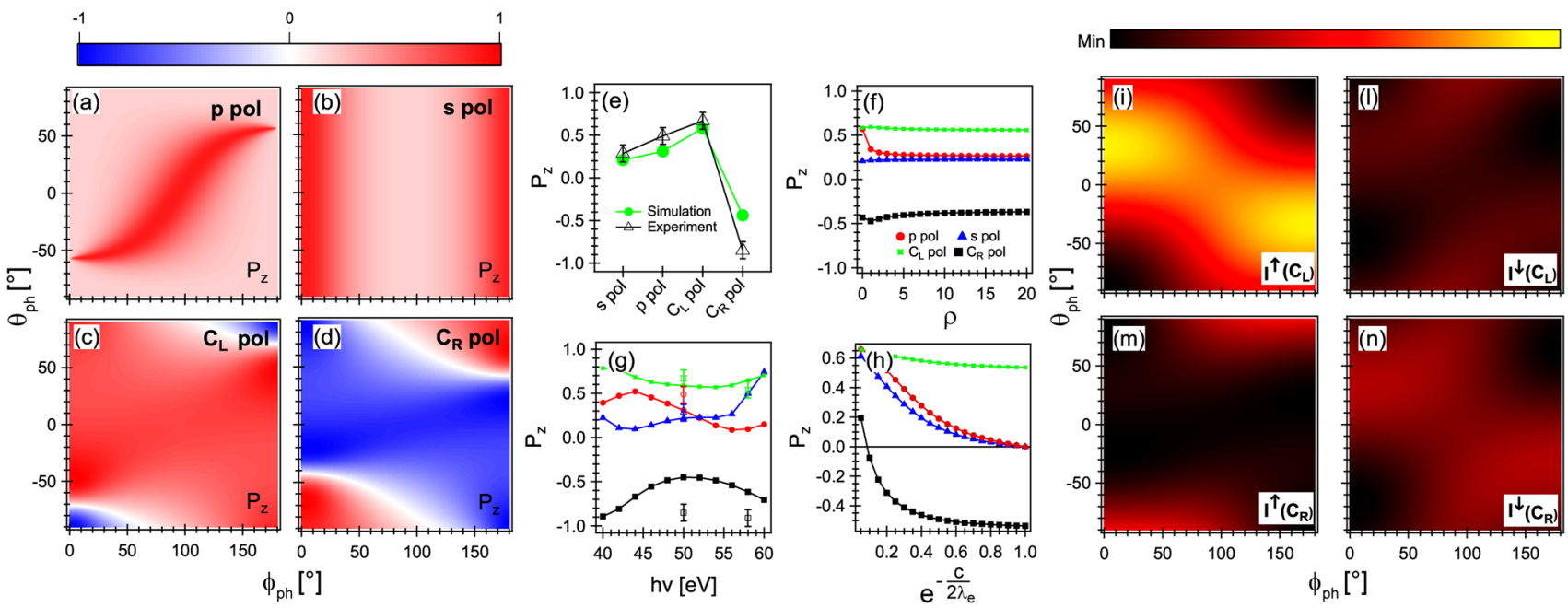

FIG. 4. Three-step model photoemission calculations. (a)-(d) Spin polarization $\left(P_{z}\right)$ maps for $p, s, C_{L}$, and $C_{R}$ polarization, respectively $(h \nu=50 \mathrm{eV})$. (e) Experimental and simulated spin polarization along $z$. (f)-(h) radial integral $(\rho)$, photon energy $(h \nu)$ and IMFP $\left(\lambda_{e}\right)$ dependence of $P_{z}$, respectively. Empty symbols in (g) show the experimental $P_{z}$ from Fig. 2(a)-(e). (i)-(n) Spin-resolved intensity maps for $C_{L}$ (top panels) and $C_{R}$ (bottom panels) polarized light.

of the uppermost layer as introduced by using linearly polarized light $[8,9,27,28]$.

This interpretation is confirmed by calculating the spinresolved ARPES intensity and the spin polarization for 8 $\mathrm{MoS}_{2}$ layers as a function of both the light geometry and polarization and using the spin doublet of the topmost VB (VB1) in valley $K$ as the initial state [11]. The spin-up (-down) state is highly localized on the odd (even) Mo layer, in analogy to the bilayer model and $\lambda_{e}$, included following the methods proposed by Refs. [22,23], has been fixed to the interlayer distance $(6.1 \AA)$.

The spin polarization intensity maps as a function of the incoming light geometry and polarization [Figs. 4(a)-4(d)] [29], show that for $s$ - and $p$-polarized light the $z$ component of the spin is positive irrespective of the geometry, in agreement with our experimental observations and with what is expected in $\mathrm{MoS}_{2}$ as the result of the finite probing depth of the photoemission process [8,9]. Our model further predicts positive and negative spin polarization for $C_{L^{-}}$and $C_{R^{-}}$-polarized lights for most of the possible experimental geometries, including ours $\left(\theta_{\mathrm{ph}}=48.3^{\circ}\right.$ and $\phi_{\mathrm{ph}}=71^{\circ}$ ), as well as sign inversion for extreme geometries corresponding to grazing incidence of the radiation [Figs 4(c) and 4(d)].

Overall, the theoretical $P_{z}$ values obtained for the various light polarization and corresponding to our geometry agree well with the experimental data as shown in Fig. 4(e). The weak dependence of the spin polarization on the radial integral of the overlap between the initial and the final state $(\rho)$ indicates that the calculations are not strongly dependent on the numerical details of the chosen states [Fig. 4(f)], and the observed spin flip is predicted to be robust against changes in the photon energy [Fig. 4(g)], as experimentally observed [Fig. 2(b)-2(e)]. The dependence of the calculated $P_{z}$ on $\lambda_{e}$ [Fig. 4(h)] also confirms that using circularly polarized light allows overcoming the IMFP limitation in variance with the case of linearly polarized lights where a presence of a small $\lambda_{e}$ is essential for observing any spin polarization. Finally, the spin-resolved intensity maps in Figs. 4(i)-4(n) account for the selective ability of the circularly polarized light to probe the layer-locked spinpolarized states for most of the possible light geometries. As the result of the finite $\lambda_{e}$ at $h \nu=50 \mathrm{eV}$, they also show higher ARPES intensities for $C_{L}$ than for $C_{R}$ [Figs. 4(i)-4(n), and 4(l) and 4(m)], with a theoretical $I^{\uparrow}\left(C_{L}\right) / I^{\downarrow}\left(C_{R}\right)$ ratio of 2.65 , in close agreement with the experimental value of $2.1[30]$.

We finally note that putative interference effects between distinct degenerate initial states [31] and/or small correction to Eq. (1), resulting from possible modification within the $\mathrm{MoS}_{2}$ monolayer, such as mirror symmetry breaking and VB1-VB2 intervalence mixing, might contribute to the spin polarization. In bulk TMDCs, these contributions only slightly change $P_{z}$ but may substantially affect the in-plane spin-polarization $\left(P_{\|}\right)$[11]. Similar to previous studies [9], we observe a small $P_{\|}$[Figs. 1(c) and 1(d)], which becomes more sizable with other polarization [11]. For supported TMDC layers the interaction with the substrate may in some cases enhance these effects whose contributions to both $P_{z}$ and $P_{\|}$might become more important [32].

In conclusion, our ARPES and spin-ARPES measurements of bulk centrosymmetric $2 \mathrm{H}-\mathrm{MoS}_{2}$ have revealed a large out-of-plane spin polarization with sign depending on the handedness of incident circularly polarized light. Our calculations, based on a three-step model of the photoemission process demonstrate that this relates to an initialstate effect of photoemission through the dipole selection rules that allow us to intimately probe the valley and layer 
spin texture of the electronic states. Our finding provides a novel route for studying hidden spin-polarized bands of inversion symmetric systems in laser or soft x-ray ARPES where the inelastic mean free path of photoelectrons is typically large.

We acknowledge F. Boschini, M. Michiardi, and A. Damascelli for valuable discussions. This project was supported by the Fonds National Suisse pour la Recherche Scientifique through Div. II.E. R. acknowledges support from the Swiss National Science Foundation (SNSF) Grant No. P300P2_164649.

*Corresponding author.

elia.razzoli@unifr.ch

Present address: Quantum Matter Institute, Department of Physics and Astronomy, University of British Columbia, Vancouver, British Columbia V6T 1Z1, Canada.

[1] K. F. Mak and J. Shan, Photonics and optoelectronics of 2D semiconductor transition metal dichalcogenides, Nat. Photonics 10, 216 (2016).

[2] D. Xiao, G.-B. Liu, W. Feng, X. Xu, and W. Yao, Coupled Spin and Valley Physics in Monolayers of $\mathrm{MoS}_{2}$ and Other Group-VI Dichalcogenides, Phys. Rev. Lett. 108, 196802 (2012).

[3] W. Jin, P-C Yeh, N. Zaki, D. Zhang, J. T. Sadowski, A. Al-Mahboob, A. M. van der Zande, D. A. Chenet, J. I. Dadap, I. P. Herman, P. Sutter, J. Hone, and R. M. Osgood, Direct Measurement of the Thickness-Dependent Electronic Band Structure of $\mathrm{MoS}_{2}$ Using Angle-Resolved Photoemission Spectroscopy, Phys. Rev. Lett. 111, 106801 (2013).

[4] Y. Zhang, T.-R. Chang, B. Zhou, Y.-T. Cui, H. Yan, Z. Liu, F. Schmitt, J. Lee, R. Moore, Y. Chen, H. Lin, H.-T. Jeng, S.-K. Mo, Z. Hussain, A. Bansil, and Z.-X. Shen, Direct observation of the transition from indirect to direct bandgap in atomically thin epitaxial $\mathrm{MoSe}_{2}$, Nat. Nanotechnol. 9, 111 (2014).

[5] T. Eknapakul, P. D. C. King, M. Asakawa, P. Buaphet, R.-H. He, S.-K. Mo, H. Takagi, K. M. Shen, F. Baumberger, T. Sasagawa, S. Jungthawan, and W. Meevasana, Electronic Structure of a Quasi-Freestanding MoS2 Monolayer, Nano Lett. 14, 1312 (2014).

[6] N Alidoust, G. Bian, S.-Y. Xu, R. Sankar, M. Neupane, C. Liu, I. Belopolski, D.-X. Qu, J. D. Denlinger, F.-C. Chou, and M.Z. Hasan, Observation of monolayer valence band spin-orbit effect and induced quantum well states in $\mathrm{MoX}_{2}$, Nat. Commun. 5, 4673 (2014).

[7] R. Suzuki, M. Sakano, Y. J. Zhang, R. Akashi, D. Morikawa, A. Harasawa, K. Yaji, K. Kuroda, K. Miyamoto, T. Okuda, K. Ishizaka, R. Arita, and Y. Iwasa, Valleydependent spin polarization in bulk $\mathrm{MoS}_{2}$ with broken inversion symmetry, Nat. Nanotechnol. 9, 611 (2014).

[8] X. Zhang, Q. Liu, J.-W. Luo, A. J. Freeman, and A. Zunger, Hidden spin polarization in inversion-symmetric bulk crystals, Nat. Phys. 10, 387 (2014).

[9] J. M. Riley, F. Mazzola, M. Dendzik, M. Michiardi, T. Takayama, L. Bawden, C. Granerd, M. Leandersson, T. Balasubramanian, M. Hoesch, T. K. Kim, H. Takagi,
W. Meevasana, Ph. Hofmann, M. S. Bahramy, J. W. Wells, and P. D. C. King, Direct observation of spin-polarized bulk bands in an inversion-symmetric semiconductor, Nat. Phys. 10, 835 (2014).

[10] V. N. Strocov, X. Wang, M. Shi, M. Kobayashi, J. Krempasky, C. Hess, T. Schmitt, and L. Patthey, Soft$\mathrm{X}$-ray ARPES facility at the ADRESS beamline of the SLS: Concepts, technical realisation and scientific applications, J. Synchrotron Radiat. 21, 32 (2014).

[11] See Supplemental Material at http://link.aps.org/ supplemental/10.1103/PhysRevLett.118.086402 for experimental and theoretical details, which includes Refs. [1217].

[12] A. Kuc, N. Zibouche, and T. Heine, Influence of quantum confinement on the electronic structure of the transition metal sulfide $T \mathrm{~S}_{2}$, Phys. Rev. B 83, 245213 (2011).

[13] M. Hoesch, T. Greber, V. N. Petrov, M. Muntwiler, M. Hengsberger, W. Auwarter, and J. Osterwalder, Spinpolarized Fermi surface mapping, J. Electron Spectrosc. Relat. Phenom. 124 (2002) 263.

[14] P. Blaha, K. Schwarz, G. K. H. Madsen, D. Kvasnicka, and J. Luitz (WIEN2K), An Augmented Plane Wave + Local Orbitals Program for Calculating Crystal Properties, edited by K. Schwarz (Technische Universität Wien, Austria, 2001), ISBN 3-9501031-1-2.

[15] G.-B. Liu, W.-Y. Shan, Y. Yao, W. Yao, and D. Xiao, Threeband tight-binding model for monolayers of group-VIB transition metal dichalcogenides, Phys. Rev. B 88, 085433 (2013).

[16] F. Meier, V. Petrov, H. Mirhosseini, L. Patthey, J. Henk, J. Osterwalder, and H. J. Dil, Interference of spin states in photoemission from $\mathrm{Sb} / \mathrm{Ag}(111)$ surface alloys, J. Phys. Condens. Matter 23, 072207 (2011).

[17] M. Hamzavi, M. Movahedi, K.-E. Thylwe, and A. A. Rajabi, Approximate analytical solution of the yukawa potential with arbitrary angular momenta, Chin. Phys. Lett. 29, 080302 (2012).

[18] K. Momma and F. Izumi, VESTA 3 for three-dimensional visualization of crystal, volumetric and morphology data, J. Appl. Crystallogr. 44, 1272 (2011).

[19] D. W. Latzke, W. Zhang, A. Suslu, T.-R. Chang, H. Lin, H.-T. Jeng, S. Tongay, J. Wu, A. Bansil, and A. Lanzara, Electronic structure, spin-orbit coupling, and interlayer interaction in bulk $\mathrm{MoS}_{2}$ and $\mathrm{WS}_{2}$, Phys. Rev. B 91, 235202 (2015).

[20] F. Meier, H. Dil, J. Lobo-Checa, L. Patthey, and J. Osterwalder, Quantitative vectorial spin analysis in angleresolved photoemission: $\mathrm{Bi} / \mathrm{Ag}(111)$ and $\mathrm{Pb} / \mathrm{Ag}(111)$, Phys. Rev. B 77, 165431 (2008).

[21] C. Jozwiak, C-H. Park, K. Gotlieb, C. Hwang, D-H. Lee, S. G. Louie, J. D. Denlinger, C. R. Rotundu, R. J. Birgeneau, Z. Hussain, and A. Lanzara, Photoelectron spin-flipping and texture manipulation in a topological insulator, Nat. Phys. 9, 293 (2013).

[22] Z.-H. Zhu, C. N. Veenstra, G. Levy, A. Ubaldini, P. Syers, N. P. Butch, J. Paglione, M. W. Haverkort, I. S. Elfimov, and A. Damascelli, Layer-by-Layer Entangled Spin-Orbital Texture of the Topological Surface State in $\mathrm{Bi}_{2} \mathrm{Se}_{3}$, Phys. Rev. Lett. 110, 216401 (2013). 
[23] Z.-H. Zhu, C. N. Veenstra, S. Zhdanovich, M. P. Schneider, T. Okuda, K. Miyamoto, S.-Y.Zhu, H. Namatame, M. Taniguchi, M. W. Haverkort, I. S. Elfimov, and A. Damascelli, Photoelectron Spin-Polarization Control in the Topological Insulator $\mathrm{Bi}_{2} \mathrm{Se}_{3}$, Phys. Rev. Lett. 112, 076802 (2014).

[24] J. Sánchez-Barriga, A. Varykhalov, J. Braun, S.-Y. Xu, N. Alidoust, O. Kornilov, J. Minár, K. Hummer, G. Springholz, G. Bauer, R. Schumann, L. V. Yashina, H. Ebert, M. Z. Hasan, and O. Rader, Photoemission of $\mathrm{Bi}_{2} \mathrm{Se}_{3}$ with Circularly Polarized Light: Probe of Spin Polarization or Means for Spin Manipulation?, Phys. Rev. X 4, 011046 (2014).

[25] C-H. Park and S. G. Louie, Spin polarization of Photoelectrons from Topological Insulators, Phys. Rev. Lett. 109, 097601 (2012).

[26] Z. Gong, G.-B. Liu, H. Yu, D. Xiao, X. Cui, X. Xu, and W. Yao, Magnetoelectric effects and valley-controlled spin quantum gates in transition metal dichalcogenide bilayers, Nat. Commun. 4, 2053 (2013).

[27] M. Gehlmann, Irene Aguilera, G. Bihlmayer, E. Młyńczak, M. Eschbach, S. Döring, P. Gospodarič, S. Cramm, B. Kardynał, L. Plucinski, S Blügel, and C. M. Schneider, Quasi 2D electronic states with high spin-polarization in centrosymmetric $\mathrm{MoS}_{2}$ bulk crystals, Sci. Rep. 6, 26197 (2016).
[28] For the case of normal incident linearly-polarized light all states with $m_{k}-m=0, \pm 1$ are equally accessible, this result in $\left|\boldsymbol{\kappa}_{2,-2} \cdot \boldsymbol{\epsilon}_{p / s}\right| \approx\left|\boldsymbol{\kappa}_{2,2} \cdot \boldsymbol{\epsilon}_{p / s}\right|$, and a positive spin polarization is observable only when a disproportion of intensity from the two initial states is introduced by the IMFP.

[29] The $P_{z}$ maps have been calculated according to the formulas $P=\left(I^{\uparrow}-I^{\downarrow}\right) /\left(I^{\uparrow}+I^{\downarrow}\right), I^{\sigma}=I^{\sigma}(1)+I^{\sigma}(2)$, where the total photoemission intensity $I^{\sigma}$ associated with a dipole transition to a final state of spin $\sigma$ contains the contributions of the two degenerate initial states $I^{\sigma}(1)$ and $I^{\sigma}(2)$.

[30] In case of finite $\lambda_{e}$ the magnitude of $I^{\uparrow}$ is similar for $s, p$, and $C_{L}$ polarizations since it is mainly determined by the photoemission from the first layer. The magnitude of $I^{\downarrow}$ for $C_{R}$ is reduced by a factor $\sim e^{-\left(c / 2 \lambda_{e}\right)}$, since it is mainly determined by the photoemission from the second layer.

[31] U. Heinzmann and J. Hugo Dil, Spin-orbit-induced photoelectron spin polarization in angle-resolved photoemission from both atomic and condensed matter targets, J. Phys. Condens. Matter 24, 173001 (2012).

[32] S.-K. Mo, C. Hwang, Y. Zhang, M. Fanciulli, S. Muff, J. H. Dil, Z.-X. Shen, and Z. Hussain, Spin-resolved photoemssion study of epitaxially grown $\mathrm{MoSe}_{2}$ and $\mathrm{WSe}_{2}$ thin films, J. Phys. Condens. Matter 28, 454001 (2016). 